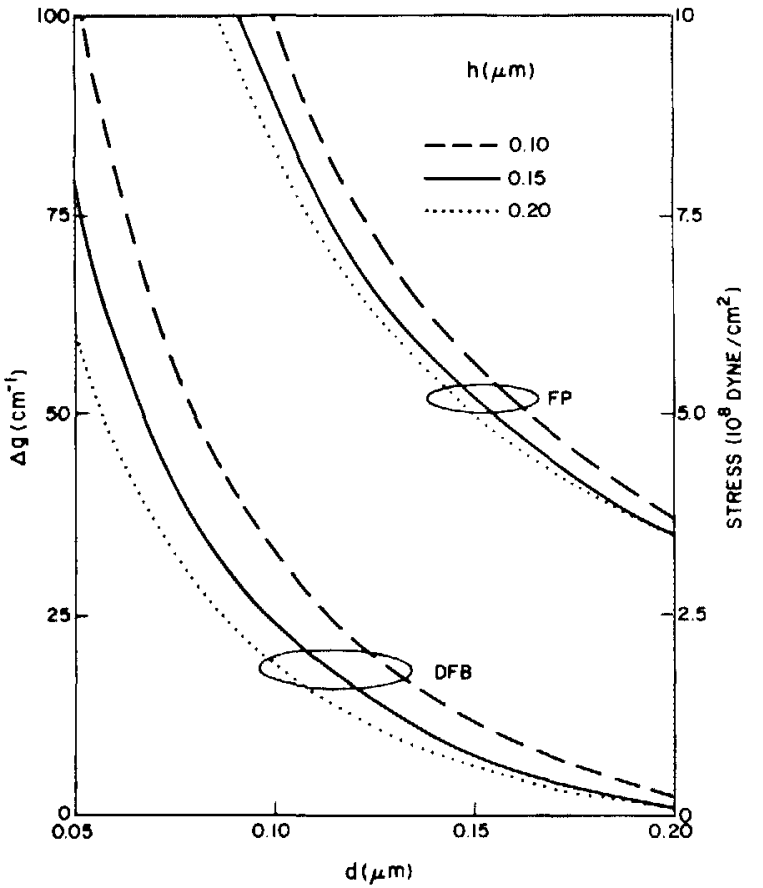

FIG. 3. Calculated gain difference $\Delta g=g_{2}-g_{1}$ and stress at which the transition from TE to TM mode occurs. Different curves correspond to different thicknesses of the anti-meltback layer. The results for a FB laser are also shown for comparison.

tice mismatch (strain) $\Delta a / a<10^{-4}$, if we assume that the elastic modulus $\sim 10^{12}$ dyne $/ \mathrm{cm}^{2}$ for InGaAsP is about the same as for GaAs. ${ }^{15}$ Figure 3 also shows that, other things equal, a thinner anti-meltback layer pushes the TM mode transition towards higher stress values.

In conclusion, we have shown that DFB semiconductor lasers have a lower TE-TM threshold margin than the conventional FP lasers and are therefore more susceptible to kink in the light-current curve related to the TM mode onset. The TE mode power at which the kink occurs can be pushed towards higher values by optimizing the active layer thickness. The numerical results are presented using parameters appropriate for a $1.55-\mu \mathrm{m} \mathrm{DC} \mathrm{PBH} \mathrm{laser.} \mathrm{For} \mathrm{devices} \mathrm{with}$ small internal stress normal to the active layer ( $\leqslant 10 \mathrm{~atm})$, a thin active layer $(d \simeq 0.1 \mu \mathrm{m})$ is preferable. On the other hand, under heavy internal stress (> $100 \mathrm{~atm}), d \simeq 0.2 \mu \mathrm{m}$ provides significant threshold margin with the TM mode reaching threshold first. The analysis is in qualitative agreement with our preliminary experimental observations. It is useful for device optimization and can be used to estimate internal stress.

The authors are thankful to P. J. Anthony, R. W. Dixon, C. H. Henry, and R. T. Yen for helpful discussions.

${ }^{1}$ H. C. Casey, Jr. and M. B. Panish, Heterostructure Lasers (Academic, NY, 1978), Chap. 2.

${ }^{2}$ N. B. Patel, J. E. Ripper, and P. Brosson, IEEE J. Quantum Electron. QE9, $338(1973)$.

${ }^{3}$ T. L. Paoli, IEEE J. Quantum Electron. QE-11, 489 (1975).

${ }^{4}$ K. G. Kalandarishvili, S. Yu. Karpov, V. I. Kuchinskii, M. N. Mizerov, E. L. Portnoi, and V. B. Smirnitskii, Sov. Phys. Tech. Phys. 28, 959 (1983).

${ }^{5}$ N. K. Dutta, J. Appl. Phys. 55, 285 (1984); N. K. Dutta and D. C. Craft, J. Appl. Phys. 56, 65 (1984).

${ }^{\circ} \mathrm{K}$. Aiki, M. Nakamura, and J.-I Umeda, IEEE J. Quantum Electron. QE$12,597(1976)$ and other references cited therein.

${ }^{7}$ K. Saki, K. Utaka, S. Akiba, and Y. Matsushima, IEEE J. Quantum Electron. QE-18, 1272 (1982) and other references cited therein.

${ }^{8}$ Y. Suematsu, S. Arai, and K. Kishino, IEEE J. Lightwave Tech. LT-1, 161 (1983).

${ }^{9}$ M. Yamaguchi, M. Kitumura, I. Mito, S. Murata, and K. Kobayashi, Electron. Lett. 20, 233 (1984).

${ }^{10}$ L. D. Westbrook, A. W. Nelson, P. J. Fiddyment, and J. S. Evans, Electron. Lett. 20, 225 (1984).

"G. L. Bir and G. E. Pikus, Symmetry and Strain Induced Effects in Semiconductors (Wiley, NY, 1974), pp. 300-322.

${ }^{12}$ H. Kogelnik and C. V. Shank, J. Appl. Phys. 43, 2327 (1972).

${ }^{13}$ W. Streifer, D. R. Scifres, and R. D. Burnham, IEEE J. Quantum Electron. QE-11, 867 (1975).

${ }^{14}$ R. F. Kazarinov and C. H. Henry, IEEE J. Quantum Electron. to be published).

15. Adachi, J. Appl. Phys. 53, 8775 (1982).

\title{
Acousto-electro-optic light modulation
}

\author{
Demetri Psaltis, Hyuk Lee, and Gabriel Sirat \\ California Institute of Technology, Pasadena, California 91125
}

(Received 24 September 1984; accepted for publication 12 November 1984)

The acousto-electro-optic effect is analyzed and demonstrated experimentally. The application of the acousto-electro-optic effect to light modulation and deflection is discussed.

The most widely used methods for wideband light modulation are either the acousto-optic $(\mathrm{AO})^{1}$ or the electrooptic $(E O)^{2}$ effects. Each type of modulator has its own strengths and suffers from its own distinct limitations. ${ }^{3}$ When we use $\mathrm{AO}$ and EO effects simultaneously, ${ }^{4}$ the acousto-electro-optic (AEO) effect, a new flexibility is intro- duced with which we can overcome some of the limitations of the two individual modulators.

The basic configuration of an AEO modulator is shown in Fig. 1. The device is operated in the normal configuration of an anisotropic $\mathrm{AO}$ modulator. A single frequency acoustic wave is launched in the crystal by the piezoelectric trans- 


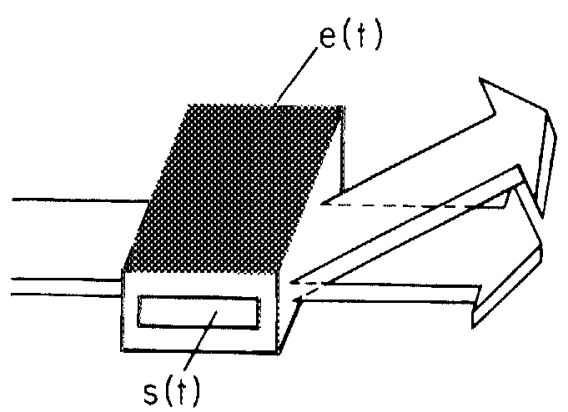

FIG. 1. Basic configuration of the AEO device.

ducer and the device is illuminated at the Bragg angle corresponding to the given frequency. The $\mathrm{AO}$ interaction is analyzed by coupled mode analysis. ${ }^{5}$ At the Bragg angle, the diffracted light intensity is maximum. If there is a phase mismatch $\Delta K$, the diffracted light intensity $I_{d}$ is given by (the approximation $\eta<1$ )

$$
\begin{aligned}
I_{d} & =\eta I_{\text {in }}\left\{\operatorname{sinc}\left[\eta+(\Delta K L / 2)^{2}\right]^{1 / 2}\right\}^{2} \\
& \simeq \eta I_{\text {in }}[\operatorname{sinc}(\Delta K L / 2)]^{2},
\end{aligned}
$$

where $I_{\text {in }}$ is the incident light intensity, $\eta$ is the diffraction efficiency of the AO interaction, $L$ is the interaction length, and $\operatorname{sinc}(x)=[\sin (x)] / x$. In the AEO device electrodes are deposited on the crystal, so that an external electrical field can be applied in addition to the acoustic wave. The application of the external field causes a deformation of the index ellipsoid due to the EO effect, and the wave-normal surfaces move accordingly. Consequently, a phase mismatch $\Delta K$ is introduced and the diffracted light intensity varies according to the amplitude of the external voltage. The phase mismatch introduced by the anisotropic AEO effect is given by

$$
\Delta K \simeq\left(\frac{2 \pi}{\lambda}\right)\left[\Delta n_{2}-\left(\frac{n_{1}}{n_{2}}\right) \Delta n_{1}\right] \simeq\left(\frac{2 \pi}{\lambda}\right) \Delta n,
$$

where $\lambda$ is the wavelength of the light in vacuum, $n_{1}$ and $n_{2}$ are indices of refraction without voltage, $\Delta n_{1}$ and $\Delta n_{2}$ are the changes of $n_{1}$ and $n_{2}$ due to EO effect, and $\Delta n=\Delta n_{2}-\Delta n_{1}$. The phase mismatch of the isotropic AEO effect is very small compared with that of anisotropic AEO effect because in the isotropic case, $n_{1}=n_{2}$ and $\Delta n_{1}=\Delta n_{2}$, and hence $\Delta K \simeq 0$. For the anistropic case we can write $\Delta n$ as follows ${ }^{2}$ :

$$
\Delta n \simeq\left[\left(n_{1}^{3} r_{1}-n_{2}^{3} r_{2}\right) / 2\right] V / h,
$$

where $h$ is the height of the crystal, $V$ is the applied voltage, and $r_{1}$ and $r_{2}$ are EO coefficients. We define the voltage $V^{\mathrm{AEO}}$ as a measure of the electrically induced phase mismatch in the AEO effect, satisfying the following:

$$
(\Delta K L / 2)=\left(\pi V / V^{\mathrm{AEO}}\right) \text {. }
$$

From equations $(2-14)$ we have

$$
V^{\mathrm{AEO}} \simeq(\lambda h / L)\left[\left(n_{1}^{3} r_{1}-n_{2}^{3} r_{2}\right) / 2\right\}^{-1} .
$$

$V^{\mathrm{AEO}}$ is approximatively equal to twice the half-wave voltage of the same crystal utilized as a transverse EO modulator. We can rewrite (1) in terms of $V^{\mathrm{AEO}}$ as follows:

$$
I_{d}=I_{\text {in }} \eta\left\{\operatorname{sinc}\left[\eta+\left(\pi V / V^{\mathrm{AEO}}\right)^{2}\right]^{1 / 2}\right\}^{2} .
$$

This is the diffracted light intensity formula of the AEO interaction and we can see that this is a direct effect, i.e., the modulation function in Eq. (6) is not simply the product of

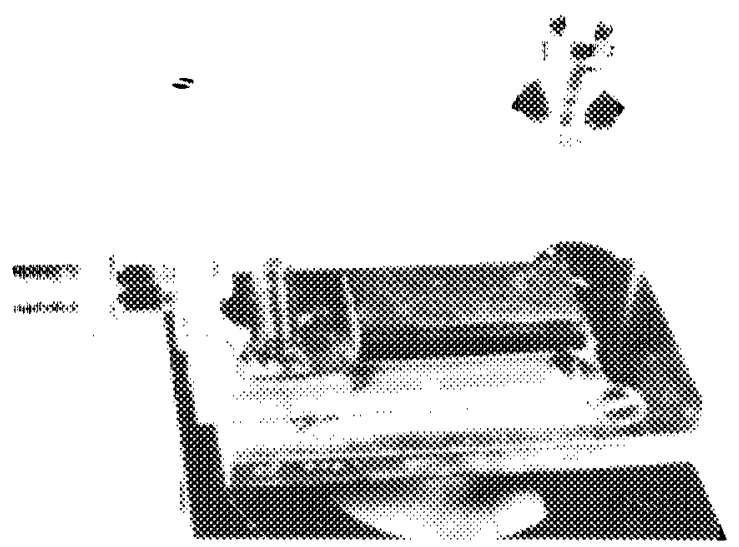

FIG. 2. Photograph of the experimental device. The size of the device was $50 \mathrm{~mm} \times 20 \mathrm{~mm} \times 20 \mathrm{~mm}$.

the electro-optic and acousto-optic effects.

An experimental device was constructed to demonstrate the AEO interaction. The device, shown in Fig. 2, was constructed from a $Y$-cut $\mathrm{LiNbO}_{3}$ crystal with dimensions $40 \times 7 \times 12(x-y-z) \mathrm{mm}$. Two electrodes were evaporated on the $(0,1,0)$ surfaces and connected to a high voltage power supply. An acoustic transducer, mounted on the $(1,0,0)$ surface, launches a $20-\mathrm{MHz}$, transverse, $y$-polarization, acoustic wave in the $x$ direction. The device is illuminated with a collimated beam from a He-Ne laser, polarized in the $y$ direction and propagating at an angle 1.8 (deg) (the Bragg angle) with respect to $z$ axis. The intensity of the diffracted light is plotted in Fig. 3 as function of the voltage (normalized to $V^{\mathrm{AEO}} / \pi$ ). The theoretical (solid) curve is given by Eqs. (1) and (6). In this experiment $V^{\mathrm{AEO}}$ was $6 \mathrm{kV}$ and $\eta$, the diffraction efficiency, was $2 \%$. The experimental results are in excellent agreement with the theoretical curve.

The simplest device that can be implemented with the $\mathrm{AEO}$ interaction is a light modulator in the arrangement of the experiment discussed in the above paragraph. Specifically if the acoustic signal consists of a single fixed frequency (the center frequency of the device) with constant power, then the diffracted light intensity can be modulated by varying the applied voltage across the electrodes of the device, in accordance to the curve in Fig. 3. The bandwidth of the AEO modulator is limited primarily by the maximum power supplied by the electrical dirving circuit with which the voltage is applied across the electrodes. The voltage level required for an AEO modulator is, within a geometrical factor close

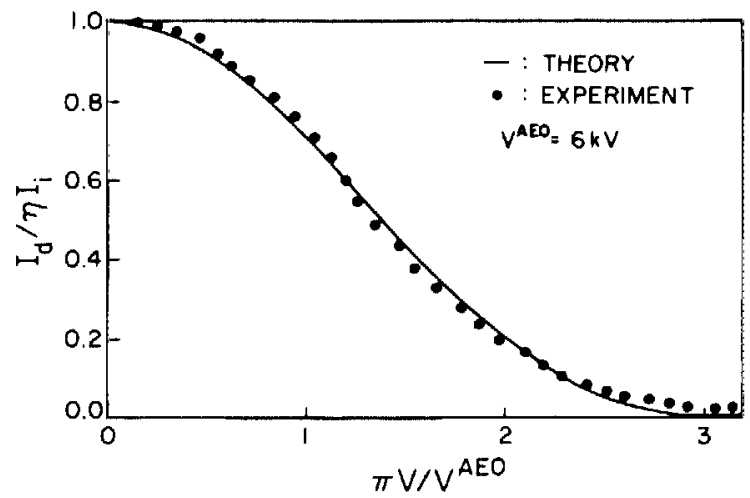

FIG. 3. Experimental results of an AEO modulator. 


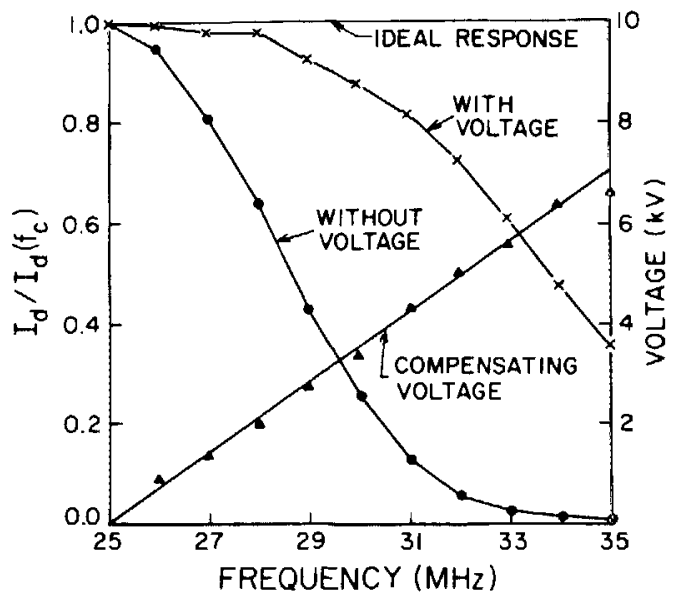

FIG. 4. Experimental results of a phase mismatch compensated AEO deflector.

to unity, twice that needed in a transverse EO modulator of the same geometry. The modulated light is angularly separated from the unmodulated light and the need for cross polarizers is eliminated. The AEO modulator can be operated with unpolarized light with a loss of diffraction efficiency of 2. The alignment of an AEO modulator (like an AO modulator) is almost insensitive to the direction perpendicular to the interaction plane. This is to be compared to the small numerical aperture, in both directions, of an EO modulator, limited by natural birefringence. The piezoelectric transducer does not affect, unlike in the AO case, the bandwidth of the modulator. Therefore, it can be designed to maximize the diffraction efficiency by increasing the transducer width and thus the interaction length. In addition the acoustic transit time limitation that is associated with the AO modulator is not encountered here and thus an extended collimated optical beam can be modulated. The acoustic port can be used to dynamically align the modulator in the plane of the interaction, by changing the center frequency, and to compensate for fluctuations in the optical input intensity by changing the intensity of the acoustic power. We have compared the linearity of the AEO and EO modulators by calculating the modulation depth obtained in the two cases when the third harmonic is set to $1 \%$ of the fundamental. The modulation efficiency is $44 \%$ in the AEO modulator and $48 \%$ in the EO modulator.

AEO interaction can also be used to improve the performance of an $\mathrm{AO}$ deflector. The numer of resolvable spots in an $\mathrm{AO}$ deflector is given by

$$
N=\Delta \vartheta_{T} / \Delta \vartheta_{0} ; \quad \Delta \vartheta_{0}=\lambda / n D,
$$

where $\Delta \vartheta_{T}$ is the total deflected angle, $\lambda$ is the optical wavelength, $n$ is the index of refraction, and $D$ is the optical aperture. $\Delta \vartheta_{T}$ is limited by the bandwidth of the deflector and determined by the transducer bandwidth and the phase mismatch introduced by the deviation of the scanning acoustic wave frequency from the center frequency. If the appropriate voltage is applied to compensate the phase mismatch introduced for each scanning frequency, a larger total deflection angle and a larger number of spots can be obtained. The phase mismatch $\Delta k$ is approximately linear in the applied voltage and for small acoustic frequency, which is the case of our experiment, it is also linear in the change of frequency $f-f_{\mathrm{c}}$. Therefore, the compensating voltage is linear in the change of frequency $f-f_{c}$. The number of spots in an AEO deflector neglecting the influence of the transducer bandwidth is given by

$$
N^{\prime}=N\left(1+2.25 V_{\max } / V^{\mathrm{AEO}}\right) \text {, }
$$

where $V_{\max }$ is the maximum voltage amplitude. The device, shown in Fig. 2, was also used to demonstrate the AEO deflector. The results of this experiment are shown in Fig. 4. The normalized intensity of the diffracted light obtained with and without the compensating voltage is plotted as a function of the acoustic frequency. The compensating voltage, as a function of the acoustic frequency, is plotted in the same figure. From Fig. 4 we can see that the bandwidth of the AEO deflector is about 2.5 larger than that of the AO deffector and was limited by the electrical bandwidth of the transducer. The compensating voltage is approximately linear in $f-f_{c}$. If the acoustic frequency is sufficiently high, so that the acoustic wavelength is comparable to the optical wavelength, we have to include a quadratic term in the relation between the phase mismatch and the frequency change; therefore, the compensating voltage will also include a quadratic term in this case.

The research reported in this paper was supported by the Air Force Office of Scientific Research.

II. C. Chang, IEEE Trans. Sonics Ultrason. SU-23, 2 (1976).

${ }^{2}$ A. Yariv, Introduction to Optical Electronics (Holt, Reinhart and Wiston, NY, 1976).

${ }^{3}$ D. Psaltis, H. Lee, and G. Sirat, Proceedings of the International Society for Optical Engineering, Los Angeles, 26-27 January 1984, Vol. 465, pp. 171-175.

${ }^{4}$ J. J. Wiczer and H. Merkelo, Appl. Phys. Lett. 30, 439 (1976).

${ }^{5}$ J. M. Rouaven, M. G. Ghazaleh, E. Bridoux, and R. Torquet, J. Appl. Phys. 50, 5472 (1979). 